FORMATION Formation emploi

Revue française de sciences sociales

96 | octobre-décembre 2006

Quand la qualification fait débat(s)

\title{
Introduction : quand la qualification fait débat(s)
}

\author{
Marie Buscatto
}

\section{OpenEdition}

\section{Journals}

Édition électronique

URL : http://journals.openedition.org/formationemploi/2496

DOI : 10.4000/formationemploi.2496

ISSN : 2107-0946

Éditeur

La Documentation française

Édition imprimée

Date de publication : 1 octobre 2006

Pagination : 5-10

ISSN : 0759-6340

\section{Référence électronique}

Marie Buscatto, «Introduction : quand la qualification fait débat(s) », Formation emploi [En ligne], 96 |

octobre-décembre 2006, mis en ligne le 14 décembre 2009, consulté le 30 octobre 2020. URL : http:// journals.openedition.org/formationemploi/2496; DOI : https://doi.org/10.4000/formationemploi.2496 


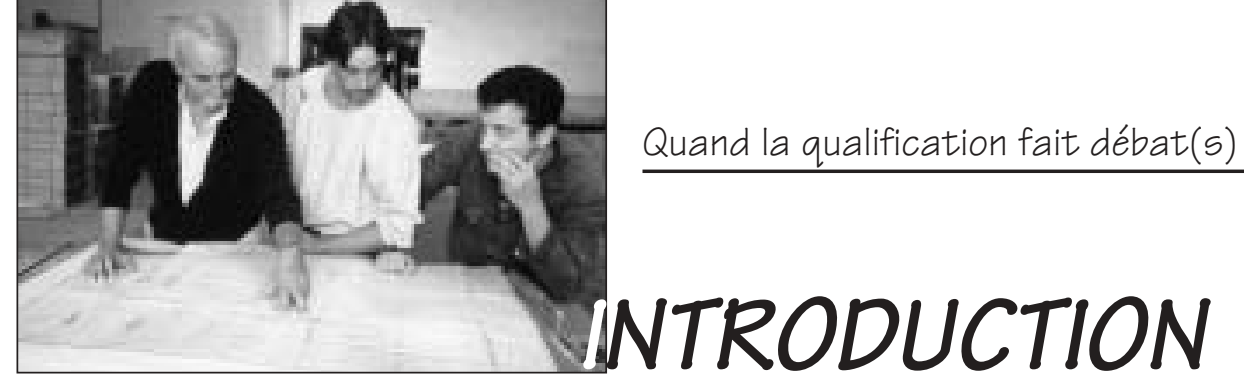

\section{Quand la qualification fait débat(s)}

Par Marie Buscatto*

Ce numéro interroge les principales logiques de qualification qui traversent les espaces professionnels contemporains à l'aube du XXIè siècle. Pour la plupart issus des Journées de sociologie du travail 2005, les articles de ce dossier s'intéressent en effet, de manière directe ou indirecte, aux modalités de reconnaissance professionnelle d'individus expérimentant des contextes professionnels bien particuliers.

Qu'il s'agisse de nouveaux emplois relationnels employé-e-s de centres d'appels (Calderón), éducateurs de centres éducatifs renforcés (Bigote), aideséducateurs (Boudesseul), ou de situations d'apprentissage particulières - doctorant-e-s (Louvel), apprenti-e-s du supérieur (Dubrion), titulaires d'un baccalauréat professionnel (Devineau)-, différentes plaintes, revendications, frustrations, négociations portées par ces travailleurs visent une meilleure reconnaissance professionnelle des savoirs mis en ouvre. Du fait des conditions ambiguës d'exercice de leur activité, des spécificités de leurs profils scolaires et/ou professionnels et des règles organisationnelles et institutionnelles dominantes, les logiques de la qualification reconnue à ces individus sont en effet source de revendications et de plaintes. Sont ainsi créés des espaces de controverses, voire de conflit pour ces travailleurs aux profils professionnels encore peu définis. Ces articles mettent alors en exergue les principaux modèles de reconnaissance professionnelle à l'œuvre dans les contextes d'emploi du XXIè siècle ainsi que les débats, controverses et compromis que leur confrontation suscite « en acte ».

Nous ferons ici un bref rappel historique des termes du débat sur la qualification professionnelle au cours des cinquante dernières années, avant d'aborder les constats énoncés à ce sujet dans les articles présentés ici.

\section{LES TERMES HISTORIQUES DU DÉBAT SUR LA QUALIFICATION AU TRAVAIL}

Un des débats constitutifs de la naissance de la sociologie du travail française porte sur la qualification au travail et s'organise autour de la célèbre opposition entre la conception «substantialiste » de Friedmann et la conception « relativiste » de Naville (CampinosDubernet M., Marry C., 1986). Dès ses origines, cette opposition se double d'une distinction implicite

\footnotetext{
* Marie Buscatto est maîtresse de conférences en sociologie au Laboratoire Georges Friedmann, université de Paris I-Panthéon-Sorbonne - CNRS. Ses travaux actuels portent sur les processus sociaux qui limitent l'accès des femmes aux professions artistiques, et plus largement aux professions socialement valorisées. De manière parallèle, elle développe une réflexion épistémologique sur l'ethnographie du travail et des organisations. Elle a notamment publié : "Des managers à la marge : la stigmatisation d'une hiérarchie intermédiaire ", Revue française de sociologie, 43 (1), 2002, pp. 73-98 ; "Chanteuse de jazz n'est point métier d'homme. L'accord imparfait entre voix et instrument en France », Revue française de sociologie, 44(1), 2003, pp. 33-60; "Ethnographies du travail artistique: apports et usages épistémologiques », Sociologie de l'art, Opus 8, 2006 , pp. 87-105.
} 
entre qualification technique et qualification sociale (Dadoy M., 1987).

L'analyse de Friedmann est définie comme «substantialiste » dans la mesure où il lui semble possible de décrire le contenu de cette qualification par l'usage de dispositifs formels ou par une observation minutieuse de l'activité de travail. Cette objectivation est alors centrée sur l'habileté professionnelle, sur le «métier » de l'ouvrier qualifié (Friedmann G., 1956). La qualification est ainsi mesurée à partir du contenu des tâches professionnelles, du contenu et de la durée de la formation professionnelle. Alain Touraine suit un même raisonnement dans ses travaux sur l'évolution du travail ouvrier (Touraine A., 1955). Il caractérise en effet trois phases dans cette évolution : phase A ou « système professionnel », phase $\mathrm{B}$ ou « système technique » et phase $\mathrm{C}$ ou «système social de production ». La phase $\mathrm{C}$ suppose que la qualification de l'ouvrier est moins définie par ses connaissances et savoir-faire techniques que par des traits de personnalité qu'il appelle alors «qualification sociale».

Pierre Naville remet en cause cette définition «substantialiste» de la qualification en la définissant comme une opération sociale de classement entre des individus dans la mesure où « il est indubitable que l'estimation purement technique de cette valeur est fallacieuse, et que c'est une estimation économique (en salaires, profits et revenus) qui s'y substitue le plus souvent; estimation elle-même coiffée par des évaluations sociales de portée plus vaste. » (Naville P., 1956, p. 130) Mais cette conception «relativiste» de la qualification reste minoritaire et peu mobilisée par les sociologues du travail jusque dans les années 90 . Alors qu'à la qualification technique ou professionnelle les chercheur-e-s tendent à associer une valeur objective et équitable en relation avec le modèle artisanal, la qualification sociale tend à être présentée comme l'expression d'une "personnalité », de traits de caractère «naturels » jugés éminemment subjectifs.

Le débat s'est prolongé dans les années 90, autour de l'opposition entre qualification et compétence. Claude Dubar montre ainsi clairement cette filiation dans un article de synthèse où il explique qu'on trouve « dans la définition tourainienne de la "qualification sociale", la plupart des traits de ce que les experts appelleront "compétence", à la fin des années 80, lorsque l'offensive $d u$ " management social " aura imposé ce terme, à la place de celui de qualification, en étroite relation avec celui de "compétitivité" (Cannac, 1986). » (Dubar C., 1996, p. 182) Seraient ainsi opposées la qualification, validation objective de savoirs techniques et théoriques via le diplôme, l'examen, l'ancienneté, le poste de travail - et les compétences, décrites comme un outil d'individualisation subjective portant sur des qualités personnelles, des possibilités propres à chaque individu (Berton F., 2006). Dans le même ordre d'idées sont opposées les compétences professionnelles, qui seraient extérieures à l'individu et susceptibles de formations techniques, de valorisations collectivement organisées, et les compétences relationnelles les fameux « savoir-être $»^{1}$ des formateurs - qui relèveraient de la personnalité, de l'intimité de l'être, de l'individualité. Les compétences relationnelles seraient l'objet d'une violence symbolique exercée sur l'intimité de l'individu et d'individualisation des jugements, jusqu'à l'exclusion des catégories les plus fragiles socialement (Dubar C., 1996; Dugué E., 1994). En revanche, la qualification, définie à partir de savoirs techniques ou théoriques mobilisés, serait à la fois le fruit d'apprentissages techniques ou scolaires objectifs et la validation de stratégies collectives de négociations syndicales.

Ce retour rapide sur les débats historiques suscités par les notions de qualification - professionnelle et sociale - et de compétence nous permet de resituer les textes de ce numéro autour de trois constats plus larges sur la qualification au travail dans la société contemporaine : la mise en concurrence des modèles de qualification dans les espaces de travail observés ; la négociation sociale comme moteur des arbitrages au sein de ces espaces professionnels; l'appel fait aux qualités «personnelles» comme modèle de qualification toujours plus prégnant et légitime.

\section{LA MISE EN CONCURRENCE DES MODĖLES DE QUALIFICATION}

Le texte de l'économiste Benjamin Dubrion permet une mise en miroir de ce constat. En s'intéressant aux seuls dispositifs formels d'évaluation de la qualifica-

\footnotetext{
${ }^{1}$ La distinction entre savoir, savoir-faire et savoir-être est largement usitée dans les milieux professionnels (formateurs, directions des ressources humaines...).
} 
tion des apprenti-e-s du supérieur en situation de travail, l'auteur fait apparaitre trois logiques de qualification idéales-typiques - qu'il appelle des «dispositifs d'évaluation du travail $»^{2}$. Ayant étudié vingt-huit grilles utilisées dans des formations du supérieur dans la région Rhône-Alpes, il fait ainsi apparaître la diversité organisée des grilles d'évaluation. Une majorité d'entre elles s'organise de manière principale autour de la logique du « savoir-être », des " compétences transversales ou comportementales». Mais ce dispositif, s'il est bien majoritaire, n'a pas supplanté d'autres logiques plus anciennes que constituent deux autres modèles de qualification: le premier porte sur les objectifs et les missions accomplis en situation de travail, alors que le deuxième est relatif aux «savoir-faire» qui "cherchent à coller aux métiers » censés être appris dans ces contextes professionnels. Si l'auteur ne montre guère comment s'établissent ces dispositifs et les éventuelles controverses qui s'établissent entre les décideurs autour de l'adoption de l'un ou l'autre modèle de qualification, il met bien en exergue les différences structurantes entre ces trois modèles de qualification professionnelle « du bon apprenti du supérieur » et leur mise en concurrence «naturelle ».

On retrouve ces débats en acte dans plusieurs cas empiriques présentés, notamment autour de l'opposition entre une logique du diplôme et du concours d'un côté et une logique des « compétences » d'un autre. Par exemple, les aides-éducateurs recrutés par l'Éducation nationale sont bien employés par leurs collègues et la direction des établissements pour assurer certaines tâches d'encadrement des élèves (soutien des élèves en difficulté, formation à l'informatique ou surveillance des enfants pendant le temps scolaire). Mais, si l'on en croit Gérard Boudesseul, ce personnel au statut temporaire et fragile peine à se voir reconnaître des compétences effectives et se trouve, selon ses dires, confronté à une «dépossession» continue des savoirs construits dans l'action. Vécues comme «le temps d'un purgatoire », ces cinq années professionnelles s'accompagnent d'un déni constant des savoirs ainsi mobilisés. Ces «gens sans qualités » se tournent alors vers le concours, seule issue professionnelle légitime à cette longue expé-

${ }^{2}$ Sont mises entre guillemets et en italique les expressions relevées dans les textes des auteur-e-s discuté-e-s. rience professionnelle peu valorisée. Dans cette administration publique, la logique du diplôme et du concours règne sans partage malgré les demandes répétées de ces acteurs, au moins lors des entretiens, pour que leur soient reconnus des «compétences», des « expériences », des « savoirs » spécifiques.

Autre exemple qui suit une même logique, celui des doctorant-e-s en sciences expérimentales étudié-e-s par Séverine Louvel. Si ces derniers sont bien considérés par les acteurs statutaires comme «la pierre angulaire du travail scientifique »-dans les discours comme dans les rapports d'activité officiels -, leur statut reste celui d'un-e étudiant-e qui deviendra, peut-être, un-e futur-e collègue, une fois la thèse et le concours obtenus. La reconnaissance professionnelle suit ici encore la logique statutaire encore dominante dans le système public, mais elle n'empêche pas qu'ici ou là émergent des revendications des unes et des autres à se voir reconnaître des « compétences» professionnelles à différents moments de ce long cursus d'apprentissage.

On voit dans ces deux exemples que, même dans des contextes institutionnels qui continuent à valoriser de manière principale une logique de qualification scolaire, les sources de revendications se développent, de manière encore très officieuse, autour de logiques de qualification concurrentes davantage centrées sur les savoirs expérientiels - professionnels et/ou relationnels - qui pourraient, à terme, se voir reconnaître une force plus prégnante. Mais quels seraient alors les ressorts d'une telle reconnaissance de savoirs encore « invisibles »? (Stroobants M., 1994)

\section{LA QUALIFICATION, UNE RECONNAISSANCE SOCIALEMENT CONSTRUITE}

Le cas des éducateurs des centres éducatifs renforcés étudiés par Capucine Bigote montre en effet que l'absence de reconnaissance d'une qualification fondée sur l'expérience et les savoirs n'est pas incontournable dans les administrations publiques. Faute de candidats répondant aux qualifications attendues, les responsables de ces centres ont dû recruter une grande majorité d'éducateurs pourtant dépourvus du diplôme $a$ priori nécessaire pour assurer cette fonction. Or, ces mêmes éducateurs, lorsqu'ils désirent construire une carrière et accroître leur rémunération au sein de cette institu- 
tion, se doivent de disposer d'un tel diplôme, seul reconnu par les politiques de gestion des ressources humaines dans le secteur social. Ils et elles vont alors obtenir des directions la construction d'un diplôme $a d$ hoc qui tout à la fois réponde à une définition classique de la qualification - savoirs théoriques validés par l'examen scolaire - et à une définition plus expérientielle de cette même qualification portée par ces nouveaux éducateurs. Ces derniers promeuvent également la Validation des acquis de l'expérience comme mode d'accès légitime à la qualification scolaire nécessaire. Ils vont, enfin, amener les acteurs traditionnels de ce secteur social à interroger les termes passés de la définition de la qualification professionnelle pour y intégrer leurs savoirs nouveaux fondés sur leur expérience.

On voit alors apparaître une différence fondamentale entre les aides-éducateurs et les doctorant-e-s d'un côté et ces éducateurs d'un autre. Quand les premiers sont en relation de forte dépendance envers leur employeur, aussi bien pour leur maintien dans l'emploi que pour la réussite de leurs éventuels projets professionnels, les éducateurs ont construit une valeur propre de leurs savoirs sur ce «marché du travail » du secteur social, obligeant les dirigeants à composer avec leurs revendications. Si dans ces trois cas, les revendications, les plaintes ou les discours de légitimation de leur professionnalité reposent bien sur une logique expérientielle peu reconnue par le système institutionnel dans lequel ils sont accueillis, les éducateurs spécialisés réussissent à faire reconnaître, au moins en partie, leur logique propre de qualification. Du fait de difficultés de recrutement de ces mêmes éducateurs et de l'efficacité supposée de leurs savoirs, ils deviennent des acteurs valorisés sur ce «marché $d u$ travail » (Paradeise C., 1987) que constituerait l'éducation spécialisée dans les centres éducatifs renforcés. Une autre logique de qualification devient alors visible, en concurrence directe avec la logique scolaire passée, et dont les contours seront redessinés afin de la rendre « acceptable » pour les acteurs statutaires. On voit ici tout l'intérêt de passer d'une définition «substantialiste» de la qualification, qui s'intéresse au seul contenu de la qualification en termes de savoir-faire, à une définition «relativiste » de la qualification qui y voit un rapport social complexe construit par les acteurs dans le temps.

C'est ce même regard "relativiste » qui permet d'ailleurs de mieux saisir le vécu paradoxal des 902 titulaires normands d'un baccalauréat professionnel étudiés par Sophie Devineau. Alors qu'ils et elles valorisent fortement leur formation pour sa capacité à les préparer à l'emploi, elles et ils sont déçus des modalités inabouties d'accès à l'emploi. S'ils sont fiers d'avoir obtenu ce titre scolaire et se sont ainsi réconciliés avec le système scolaire, ils ne trouvent guère la reconnaissance attendue dans l'enseignement supérieur - faible accès aux formations BTS (brevet de technicien supérieur) - ou dans le monde du travail - chômage ou emplois peu valorisés. Seule une analyse en termes de qualification permet ici de comprendre cet apparent paradoxe. Quand les titulaires d'un baccalauréat professionnel se considèrent détenteurs de savoirs théoriques et professionnels élevés, du fait de leur accès à ce titre scolaire et à la formation professionnelle qui l'accompagne, les employeurs ne semblent guère " voir » ces savoirs et leur attribuer la valeur attendue. La valeur sociale du diplôme diffère dans les esprits des un-e-s et des autres, créant en retour de relatives frustrations chez ces jeunes diplômé-e-s. Du fait de l'invisibilité de leurs savoirs sur le marché du travail, ces titulaires d'un baccalauréat professionnel sont perçus comme des personnes encore peu qualifiées alors qu'ils et elles ont une évaluation toute différente de leurs capacités professionnelles.

La valeur sociale attribuée aux savoirs des individus semble bien dépendre de la logique principale de qualification à l'œuvre dans les contextes organisationnel, institutionnel et social qui les accueillent. Mais l'adoption de cette logique - et son éventuelle remise en cause, au moins partielle - dépend de nombreux paramètres que nous ne pourrons ici qu'énoncer : la concurrence sur le marché du travail qui rend ces individus plus ou moins rares et difficiles à recruter ; la force et la prégnance de groupes de pression (syndicats, associations) protecteurs ; les stéréotypes sociaux qui rendent ces savoirs plus ou moins «visibles $»^{3}$; les règles institutionnelles dominantes qui privilégient telle ou telle logique de qualification;

\footnotetext{
${ }^{3}$ Pour exemple, dès les années 60, Madeleine Guilbert constatait que «les employeurs utilisent dans l'univers de la production industrielle des compétences que les femmes ont acquises dans la sphère familiale par le travail domestique. [...] Ces qualités sont donc à la fois repérées et niées: ce sont des qualités féminines dites "naturelles" et donc précisément pas des qualifications professionnelles. » (citée in Maruani M., 2000, p. 44)
} 
ou encore les caractéristiques sociales des acteurs qui les portent. En l'absence d'un accord sur les fondements même de la qualification légitime, les débats, les revendications, les oppositions se multiplient au sein des institutions certes, mais aussi dans les discours des acteurs qui peuvent alors se sentir « invisibles », «dépossédés », "dévalorisés », « dénigrés »... La qualification est un rapport économique socialement construit, fruit d'une incessante négociation entre des acteurs aux intérêts divergents.

\section{LA PRÉGNANCE TOUJOURS PLUS FORTE D'UNE LOGIQUE $D E$ «PERSONNALISATION »}

Un dernier constat que nous aimerions maintenant discuter est la prégnance d'un modèle managérial centré sur la « personne » du travailleur qui apparaît soit de manière officielle - à travers les dispositifs formels mis en œuvre dans les contextes étudiés -, soit de manière informelle - à travers les comportements et les revendications des acteurs qui demandent à être reconnus en tant que " personnes ».

Benjamin Dubrion a bien saisi, dans son étude sur les «dispositifs d'évaluation du travail » des apprenti-e-s $\mathrm{du}$ supérieur en situation professionnelle, la forte prégnance d'un modèle de qualification qui valorise les «savoirs relationnels», les «compétences», les qualités «personnelles » des individus. José Calderón, dans son étude menée dans un centre d'appels du secteur de la distribution énergétique, montre comment les dirigeants en appellent constamment aux «qualités personnelles » des salarié-e-s. Des «dispositifs d'entraînement $»-$ formation formelle et informelle, coaching - sont mis en œuvre pour façonner le sourire, les discours, les comportements relationnels des individus. On retrouve ainsi le modèle managérial observé au cours des années 90 dans les grandes organisations modernes (Boltanski L., Chiappello E., 1999 ; Linhart D., 1999). Dans leur grande majorité, les sociologues du travail y voient le signe d'une " personnalisation " croissante de toutes les sphères du travail - industriel ou de service, managérial ou subordonné, relationnel ou technique. L'appel fait à «l'implication de soi » et aux « compétences personnelles» se conjugue progressivement avec la reconnaissance des savoirs théoriques ou techniques dans les contextes de travail les plus variés.
Mais les individus se révèlent aussi des acteurs actifs de la personnalisation des situations professionnelles et, paradoxalement, ils et elles deviennent ainsi porteurs du développement de la logique de personnalisation observée (Buscatto M., 2002 ; 2004). Les opérateurs téléphoniques résistent aux injonctions des directions qui ne leur semblent pas respecter leur «subjectivité » et leurs conceptions de la «bonne relation-client ». Ils peuvent ainsi soit appliquer une "émotivité de surface» afin de ne pas subir cette personnalisation des situations, soit refuser tout simplement sa mise en œuvre dans l'acte de travail. Les aides-éducateurs, tout comme les éducateurs des centres d'éducation renforcée, font preuve d'une forte personnalisation de leurs situations professionnelles et en revendiquent aussi bien la nécessité que la légitimité professionnelles. Quand les premiers voient dans leur « vécu», dans leurs " expériences » passées, dans leurs "investissements affectifs et personnels》, les causes de leur supposée efficacité en situation de travail, les deuxièmes «tendent à revendiquer diverses formes de reconnaissance, en particulier sur le plan de l'utilité, de la sociabilité, voire de la subjectivité relationnelle ». Ces acteurs attendent une reconnaissance formelle et informelle de leurs «qualités personnelles » en dehors même des dispositifs formels de reconnaissance à l'œuvre dans ces organisations.

Sans s'y référer de manière explicite, les auteur-e-s de ce dossier mettent en œuvre une conception «relativiste » de la qualification toute navillienne. Ils et elles ont en cela suivi Mateo Alaluf, pour lequel l'opération de qualification des individus ne peut être dissociée de la reconnaissance des savoirs mobilisés dans le contexte social où cette opération se réalise. Le savoir scolaire validé par un examen n'est pas plus objectif que le savoir relationnel mis en œuvre dans une relation de service, car «vouloir y (la qualification) déceler un noyau technique irréductible à sa nature sociale, n'est rien d'autre qu'une tentation de naturaliser des différences sociales et de pérenniser les formes hiérarchiques résultant des jugements sociaux en vigueur dans une société donnée. » (Alaluf M., 1992, p. 7)

\footnotetext{
${ }^{4}$ Nous avons montré ailleurs comment cette prise de position épistémologique permet de développer une analyse strictement sociologique des évolutions de la qualification dans les organisations modernes. Nous ne pouvons ici en présenter l'argumentation complète que le lecteur ou la lectrice pourra retrouver in Buscatto M., 2004.
} 
Cette posture permet ainsi, à partir d'une description précise aussi bien des situations de travail rencontrées que des revendications portées par les individus, de déceler les termes de la qualification à l'œuvre dans ces espaces professionnels. Les débats, controverses, résistances, plaintes observés entre les différents protagonistes révèlent bien en retour que plusieurs logiques de qualification sont aujourd'hui mises en concurrence dans les différents espaces professionnels. Les systèmes de qualification apparaissent comme des produits sociaux complexes, traversés certes par des logiques «idéales-typiques » structurantes, mais aussi comme les produits de négociations incessantes qui peuvent en transformer le sens.

\section{Bibliographie}

Alaluf M. (1992), «Peut-on distinguer les classements techniques des classements sociaux de la qualification? » Formation Emploi, 38, pp. 3-7.

Berton F. (2006), « Le lien compétence-individu, une relation ni directe, ni immédiate », Journée d'études Usages sociaux de la notion de "compétence»: Quels savoirs? Quels individus, Paris, CNAM, 9 mars.

Boltanski L., Chiappello E. (1999), Le nouvel esprit du capitalisme, Paris, Gallimard.

Buscatto M. (2002), « Des managers à la marge : la stigmatisation d'une hiérarchie intermédiaire », Revue française de sociologie, 43 (1), pp. 73-98.

Buscatto M. (2004), «Les savoirs relationnels : expression de "soi" ou qualification sociale?", Sociologia del Lavoro, 95, pp. 54-66.

Campinos-Dubernet M., Marry C. (1986), « De l'utilisation d'un concept empirique : la qualification ». in Tanguy L., L'introuvable relation formationemploi. Etat d'un champ de recherche en France, Paris, La Documentation française.

Dadoy M. (1987), « La notion de qualification chez Georges Friedmann », Sociologie du travail, 29 (1), pp. 15-34.

Dubar C. (1996), « La sociologie du travail face à la qualification et à la compétence », Sociologie $d u$ travail, 38 (2), pp. 179-193.
Dugué E. (1994), « La gestion des compétences : les savoirs dévalués, le pouvoir occulté », Sociologie $d u$ travail, 36 (3), pp. 273-292.

Friedmann G. (1956), Le travail en miettes, Paris, Gallimard.

Linhart D. (1999), « L'individu au cœur de la modernisation des entreprises : une reconnaissance attendue mais périlleuse » in Contributions au Colloque 40 ans de sociologie du travail, 25-26 novembre.

Maruani M. (2000), Travail et emploi des femmes, Paris, La Découverte.

Maurice M., Sellier F. et Silvestre J.-J. (1982), Politique d'éducation et organisation industrielle en France et en Allemagne : essai d'approche sociétale, Paris, PUF.

Naville P. (1956), Essai sur la qualification $d u$ travail, Paris, Librairie Marcel Rivière.

Paradeise C. (1987), « Des savoirs aux compétences : qualification et régulation des marchés du travail », Sociologie du travail, $\mathrm{n}^{\circ}$, pp. 35-46.

Stroobants M. (1994), «La visibilité des compétences » in Rope F., Tanguy L., Savoirs et compétences. De l'usage de ces notions dans l'école et dans l'entreprise, Paris, L'Harmattan, pp. 197-222.

Touraine A. (1955), L'évolution du travail ouvrier aux Usines Renault, Paris, CNRS. 